\title{
IMPLICACIONES ÉTICAS QUE ENFRENTA EL DOCENTE AL SUPERVISAR LA PRÁCTICA CLÍNICA DE ESTUDIANTES DE ENFERMERÍA
}

\section{ETHICAL IMPLICATIONS FACED BY TEACHERS SUPERVISING THE CLINICAL PRACTICE OF STUDENTS OF INFIRMARY}

Lorena Martínez Delgado ${ }^{1}$

Vilma Cenit Fandiño Osorio ${ }^{2}$

\section{RESUMEN}

Durante la práctica clínica, la Enfermera Docente se ve abocada a actuar en situaciones que implican la toma de decisiones éticas frente al paciente y de responder por las consecuencias de la decisión. El objetivo de la investigación, fue determinar las implicaciones deontológicas más frecuentes en las que se ve involucrado el profesional de Enfermería, en el ejercicio de la docencia, al supervisar la práctica clínica de estudiantes, en la ciudad de Bogotá. Son escasas las investigaciones que se encuentran al respecto, si se tiene en cuenta que es un tema que cada vez cobra mayor importancia en la práctica profesional. Como instrumento de recolección, se diseñó una entrevista semiestructurada y grabaciones de experiencias relatadas por los profesionales participantes. Los resultados determinaron que las implicaciones, a las que con mayor frecuencia se enfrentan los docentes, son de tipo ético, no se presentó ninguna denuncia penal ante el tribunal de Ética de Enfermería, en la que se hubiese

${ }^{1}$ Enfermera. Especialista en Docencia Universitaria y en Educación para la Salud. Facultad de Enfermería. Universidad de Ciencias Aplicadas y Ambientales U.D.C.A. e-mail: lorenamartinez53@hotmail.com.

${ }^{2}$ Enfermera. Especialista en Gerencia Educativa. Magister con énfasis en Cuidado Materno. Facultad de Enfermería. Universidad de Ciencias Aplicadas y Ambientales U.D.C.A. e-mail: vfandino@udca.edu.co. impuesto como sanción la destitución, el retiro de la tarjeta profesional o la indemnización de perjuicios. Las situaciones que vive el estudiante con el Sujeto de Cuidado, le generan experiencia en el razonamiento moral y en la importancia de los valores, para la toma de decisiones éticas. Se evidencia la necesidad de establecer una comunicación contínua con las Instituciones en los escenarios de práctica, que permita coordinar la atención del Sujeto de Cuidado y un consenso en la toma de decisiones, cuando la situación lo requiera.

Palabras clave: Ética, enfermería, docencia-asistencia, responsabilidad, dilema ético.

\section{SUMMARY}

During clinical practice the teaching nurse is doomed to act in situations that involve making ethical decisions in front of the patient and be responsible for the consequences of this decision. The objective of the research was to determine the more frequent implications in which the infirmary professional is involved in hospitals, while supervising the student practice in the city of Bogotá. Research on this topic is scars, taking into account that this subject becomes, every time, more and more important in the professional exercise. As a tool for data collecting, a semi-structured interview was designed and recordings of experiences by the participant professionals were made. The results determined that the implications most frequently faces by the teacher in the places of practice are of ethical type; no penal denunciation, before the court of Ethics 
of Infirmary, followed by a sanction or the dismissal, withdrawal of the professional card or indemnification of prejudices was presented. The situations confronted by students with the care subject generate experience in moral reasoning and in the importance of values for the taking of ethical decisions. The necessity to establish a continuous communication within the institutions, where the practice is realized, will allow a proper coordination of the attention of the care subject and a consensus in the decision making, when required by the situation.

Key words: Ethics, infirmary, teaching-attendance, responsibility, ethical dilemma.

\section{INTRODUCCIÓN}

Los cambios realizados en el Sistema General de Seguridad Social en Salud y las reformas laborales en Colombia han afectado las condiciones de trabajo del sector salud, específicamente, las relacionadas con la contratación, estabilidad del personal y responsabilidad dentro de las Instituciones. El aumento en la carga laboral, la reducción de personal y la vinculación temporal de los profesionales de Enfermería en Instituciones Prestadoras de Salud son factores que influyen, de manera directa, en la calidad de atención del paciente al reducirse el tiempo para brindar cuidado directo; así mismo, el personal a quien se le delega esta función muchas veces no cuenta con la experiencia necesaria para llevarla a cabo.

Otro factor que agrava esta situación, se relaciona con las políticas establecidas dentro de las instituciones concernientes a la reducción en el tiempo de estancia hospitalaria del paciente, con el propósito de reducir costos.

Estas situaciones conducen a que los profesionales de la Salud se enfrenten con mayor frecuencia a dilemas éticos, donde la toma de decisiones puede afectar la vida de los pacientes y, en muchas ocasiones, ser confrontadas con los valores morales, la ética y las prioridades de los Sujetos de Cuidado.

Para entender el marco dentro del cual se deben tomar las decisiones éticas es importante conocer algunas de las clasificaciones de las teorías éticas:

a. Teorías clásicas y modernas

b. Teorías consecuentes y no consecuentes.
En las teorías clásicas encontramos el hedonismo (el placer es la única calidad de la vida humana) y el estoicismo (la virtud como calidad principal); las teorías modernas aplican análisis filosóficos a la conducta ética, a fin de averiguar el significado de los términos y las declaraciones que aparecen en las teorías y en esta categoría, se ubican el naturalismo, el emotivismo y el intuisionismo.

Las teorías consecuentes examinan las consecuencias de los actos y afirman que una acción es apropiada en la medida en que produce consecuencias favorables y, desacertadas, si sus consecuencias son negativas. El utilitarismo es un ejemplo de este tipo de teorías (Fry, 1994).

Las teorías no consecuentes son las que mantienen que ciertos actos son buenos y otros son malos, porque poseen características que dan lugar al bien o carecen de ellas. La deontología es un tipo de teoría no consecuente, según, la cual, las acciones son acertadas si se basan en reglas o en leyes relativas a derechos u obligaciones, independientemente, de sus consecuencias o resultados (Fry, 1994).

De igual manera, todas las teorías éticas tienen principios que son pautas para la toma de decisiones morales y la acción moral. Los principios para la práctica de Enfermería son: justicia, veracidad, autonomía, beneficencia y fidelidad.

La ética profesional es un conjunto de principios que, al aplicarse en la práctica, permiten juzgar la bondad de las actividades profesionales en términos humanos y legales, y se basa en los derechos fundamentales del hombre como individuo y como miembro de una sociedad.

Para los profesionales de Enfermería, el cuidado se constituye en un acto ético que implica responsabilidad, fidelidad, sensibilidad, el respeto por la vida y la preservación de la dignidad del ser humano.

La ética del cuidado considera que no sólo incluye las actividades que realiza la enfermera sino cómo ejecuta cada uno de los actos de cuidado, es decir, la forma, los motivos por los que los realiza y las relaciones que establece con el sujeto de cuidado.

Algunos autores afirman que el Cuidado de Enfermería es el eje en torno al cual gira la práctica de enfermería, 
le da su esencia y establece la diferencia con la práctica médica. Perry E Potter (2002) citando a Noddings (1984), exponen que en la ética del cuidado se identifican "el cuidador"; para designar a aquella persona moral individual que brinda cuidado a un paciente y "el cuidado", para el sujeto que requiere un Cuidado de Enfermería.

Para realizar una toma de decisiones acertada cuando se presenta un dilema ético es necesario conocer qué significado tienen los valores en cada una de las personas y la prioridad que estos dan a cada uno de ellos, pues en los dilemas éticos, los valores de cada una de los individuos entran en juego y determina la solución en la decisión frente al suceso.

La capacidad de toma de decisiones éticas es esencial en la práctica profesional de la Enfermería. Los estudiantes deben aprender a integrar sus valores y convicciones personales con el conocimiento de conceptos éticos, enfoques tradicionales y contemporáneos hacia la ética y normas de comportamiento ético. Esta integración formará parte del marco para la toma de decisiones éticas y su aplicación en los Cuidados del Sujeto de atención (Fry, 1994).

El modelo de toma de decisiones para la diversidad ética y cultural, planteado por Fry, establece cuatro interrogantes que ayudan a comprender el contexto, donde se suscita el problema ético, el significado de los valores, el significado del problema para las partes interesadas y la determinación de lo que se debería hacer. Estos interrogantes son:

1. ¿Cuáles son los antecedentes del conflicto?

2. ¿Cuál es el significado de los valores en cuestión?

3. ¿Cuál es el significado del conflicto para las partes en cuestión?

4. ¿Qué hacer?

En la medida en que se conozcan los significados de los valores será más fácil reconocer cuáles son los valores que se deben preservar y proteger frente a la decisión que se ha de tomar.

Ante un dilema ético rara vez se puede tomar una decisión correcta o pensar que existe una única solución, lo importante, es evaluar los resultados y el procedimiento que se siguió, para la toma de la decisión (Fry, 1994).
Perry E Potter (2002), por su parte, exponen que los dilemas éticos producen tensión en las personas implicadas en el proceso y requieren de un procedimiento sistemático, conciente y prudente. Estas autoras comparan el dilema ético con el pensamiento crítico y para la resolución de estos dilemas, plantean las siguientes etapas:

Etapa 1. Identificar si se trata de un dilema ético. Si una revisión de los datos científicos no resuelve la cuestión, la cuestión es confusa y la respuesta tendrá una enorme relevancia en varias áreas del conocimiento humano, puede existir un dilema ético.

Etapa 2. Reunir toda la información relevante sobre el caso.

Etapa 3. Examinar y determinar los propios valores sobre las cuestiones. La clarificación de los valores proporciona un fundamento para la claridad y la confianza durante las controversias que serán necesarias para la resolución del dilema.

Etapa 4. Verbalizar el problema.

Etapa 5. Considerar las posibles vías de actuación.

Etapa 6. Negociar el resultado.

Etapa 7. Evaluar la actuación (Perry E Potter, 2002).

Estos modelos de toma de decisiones y de protocolos brindan un marco de referencia para enfrentar los dilemas éticos a los que se ven enfrentados los profesionales de la salud y los estudiantes en formación en las Instituciones Prestadoras de Salud.

Es importante aclarar que las intervenciones en enfermería no solamente tienen implicaciones éticas, también pueden ser de tipo legal o ambas. Las normas jurídicas y éticas, se desarrollan dentro del mismo clima histórico, social, cultural y filosófico, por tanto, es importante aclarar el significado de cada uno de estos términos.

Los individuos tienen derechos legales fundados en la ley, también tienen derechos morales arraigados en sistemas, en principios y en reglas éticas. Los derechos legales son válidos en el sistema jurídico y los derechos morales se derivan de costumbres, tradiciones e ideales (Fry, 1994).

Son escasos los estudios sobre implicaciones éticas y legales en el ejercicio profesional docente, debido 
a que las investigaciones se enfocan en temas como la inclusión de la ética y la Bioética en los currículos de Enfermería, la Bioética y el conocimiento de las implicaciones éticas y legales en las que se puede incurrir en el ejercicio profesional.

De esta situación surge la motivación para realizar la investigación, que se convierte en el objeto de este artículo, y determinar las implicaciones deontológicas del profesional de Enfermería en el ejercicio de la docencia, que supervisa la práctica clínica de los estudiantes, en la ciudad de Bogotá.

El Código Deontológico de Enfermería hace referencia al conjunto de deberes y normas éticas y morales que debe conocer el profesional de Enfermería, para aplicarlas en su quehacer diario (Ley 911, 2004).

\section{MATERIALES Y MÉTODOS}

La investigación fue de tipo descriptivo y retrospectivo. La muestra fue de 22 Enfermeras de Instituciones de Educación Superior encargadas de supervisar las prácticas clínicas de los estudiantes, en la ciudad de Bogotá. Los criterios de inclusión fueron: ser Enfermera Docente, supervisar prácticas clínicas de estudiantes y laborar en una Institución de educación superior. La participación de los profesionales fue voluntaria y con consentimiento informado.

Se emplearon como instrumentos de recolección de la información, una entrevista estructurada y grabaciones en casette, de experiencias directas, relatadas por profesionales de Enfermería, referentes a la supervisión clínica de estudiantes en formación.

La elaboración de la entrevista (Cuadro 1), se fundamentó en la Ley 266 de 1996, que reglamenta el ejercicio de la Enfermería en Colombia y establece las competencias y funciones, el Modelo decisorio relativo a la diversidad ética y cultural en la práctica de enfermería propuesto, por Fry (1994) y la Ley 911 de 2004, que en el capítulo IV, artículo 31, establece la responsabilidad del profesional de Enfermería en la investigación y en la docencia, así "El profesional de enfermería en el ejercicio de la docencia para preservar la ética en el cuidado de enfermería que brindan los estudiantes en la práctica de aprendizaje, tomará las medidas necesarias para evitar riesgos y errores que por falta de pericia ellos puedan cometer" (Ley 911, 2004).
Una vez recopilada la información, se establecieron las categorías de análisis para las preguntas abiertas, teniendo en cuenta las respuestas dadas por los Profesionales participantes en la Investigación y la frecuencia de respuesta en las preguntas de selección múltiple, predeterminadas en la entrevista.

A estos resultados, se le sacaron cifras porcentuales, teniendo en cuenta la frecuencia de respuesta de la pregunta.

\section{RESULTADOS Y DISCUSIÓN}

Los resultados de la Investigación determinaron que las implicaciones a las que con mayor frecuencia se enfrentan los docentes en los sitios de práctica son de tipo ético; no se presentó ninguna denuncia penal o quejas ante la oficina de control interno de las Instituciones Prestadoras de Salud o querella ante el tribunal de Ética de Enfermería en la que se hubiese impuesto como sanción la destitución, el retiro de la tarjeta profesional o la indemnización de perjuicios.

Al clasificar las implicaciones Éticas, se encontraron de tipo disciplinar, de recursos y de tipo administrativo (Gráfica 1).

Las implicaciones de tipo disciplinar, se relacionaron con sucesos, donde el estudiante realizó actividades de Cuidado directo sin la debida supervisión, lo que puso en riesgo el estado de salud del paciente, sin que tuviera consecuencias graves para el mismo.

Las implicaciones administrativas y de recursos fueron consecuencia del poco tiempo de estancia hospitalaria de los Sujetos de Cuidado, la disponibilidad de recurso humano y de materiales y la calidad del material, para brindar una atención adecuada y oportuna.

La falta de disponibilidad de recurso humano y material en una Institución Prestadora de Salud pone en riesgo la vida del Sujeto de Cuidado y se opone a las condiciones para el ejercicio de la Enfermería, establecidas en la Ley 911 de 2004, en el capítulo II, Artículo 5, Parágrafo: “del déficit de las condiciones para el ejercicio de la enfermería, el profesional deberá informar por escrito a las instancias de enfermería y de control de la Institución y exigirá el cambio de ellas, para evitar que esta situación se convierta 
Cuadro 1. Entrevista.

Queremos agradecer su participación en esta investigación. Solicitamos a Usted que la información que nos brinde sea lo más descriptiva y veraz posible. Su nombre no será registrado por razones de confidencialidad y su identidad es reservada.

Durante la etapa de formación en Enfermería, los estudiantes, los docentes y los profesionales que están involucrados en las prácticas profesionales que se desarrollan a lo largo de la carrera, enfrentan situaciones particulares, que pueden confrontar sus valores éticos, religiosos y morales. Estas situaciones pueden llevar a vulnerar la autonomía del paciente, los derechos del educando a aprender y, a su vez, que comprometen la responsabilidad del docente y del personal de salud.

1. ¿Usted en su práctica docente en instituciones hospitalarias se ha enfrentado a situaciones, en las cuales, sus principios deontológicos hayan sido vulnerados?
a. De tipo disciplinar
b. Administrativo
c. Gerencial
d. Recursos

2. Describa la situación, fecha y servicio en el que ocurrió el evento.

3. ¿Cómo se solucionó la situación? ¿Qué tipo de alternativas se tuvieron en cuenta? El evento se solucionó entre:
a. Docente-estudiante
b. Docente-estudiante-Jefe del servicio
c. Docente-estudiante-Jefe del servicio-Jefe del departamento
d. Docente-estudiante-Jefe del servicio-Jefe del departamento-Coordinador del Servicio
e. Docente-estudiante-Jefe del servicio-Jefe del departamento-Coordinador del Servicio-Institución de Salud.
f. Docente-estudiante-Jefe del servicio-Jefe del departamento-Coordinador del Servicio-Institución de Salud-Institución Universitaria.

4. ¿Se realizó algún análisis de riesgo, en cuanto a causas del error-factores de riesgo?
a. Falta de claridad en las responsabilidades
b. Falta de claridad en las funciones
c. Descuido
d. Ignorancia
e. Omisión

5. ¿A qué sistema de apoyo acudió para tomar la decisión frente al suceso? ¿Cuál fue la participación de las instituciones en el proceso?

6. ¿De qué dependió la toma de decisiones?, ¿Por qué?, ¿Dónde, cuándo, cómo y qué sentimiento rodearon el suceso?

7. ¿Qué decisión se tomó?

8. Para usted como Docente ¿esta situación llegó a tener implicaciones disciplinarias en la Institución donde labora?
a. Amonestación verbal o escrita
b. Suspensión de 1-5 días
c. Suspensión hasta de 30 días
d. Cancelación del contrato

9. A nivel personal ¿qué implicaciones tuvo el suceso?

10. ¿Qué implicaciones tuvo el suceso para el estudiante?

11. ¿Cree usted que hubiera podido manejarlo de otra forma, ¿cuál?

12. ¿Qué aprendizaje le quedó claro para enfrentarse a una situación similar?

13. ¿Qué esta haciendo en la actualidad? 


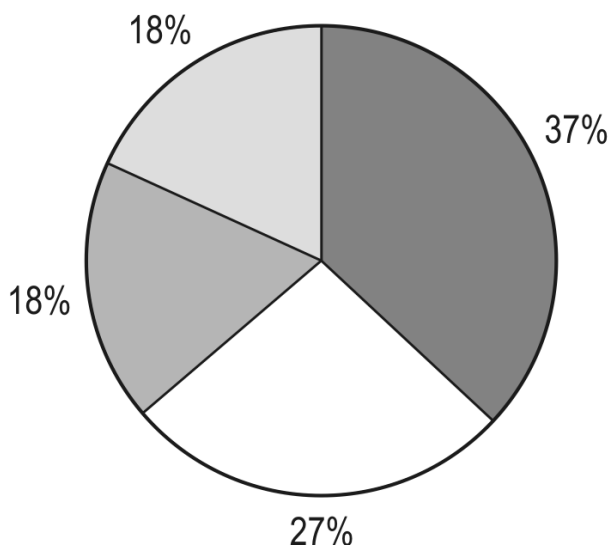

De tipo disciplinar

Recursos material o de talento humano

Administrativo o Gerencial

No se ha presentado

Gráfica 1. Clasificación de las implicaciones.

en condición permanente que deteriore la calidad técnica y humana de los servicios de enfermería".

En cuanto a causas del error, factores de riesgo, los resultados mostraron descuido frente al cuidado del paciente, falta de claridad en las funciones del personal de Enfermería y carece de comunicación entre los integrantes del equipo de salud.

Para brindar Cuidado de Enfermería se requiere de un trabajo en equipo, donde cada uno de los actores tenga claridad frente a las funciones y a las responsabilidades como profesional al ejercer su rol de cuidador.
Referente a las instancias a las que se acudió para solucionar el evento, la mayoría de las situaciones se solucionaron y se aclararon entre el personal de Enfermería de las dos Instituciones (educativa y prestadora de salud), sin que llegara a trascender a otras dependencias (Gráfica 2).

Los conflictos a los que se enfrentan los profesionales de Enfermería se ven influidos por decisiones administrativas. Uno de los roles del profesional de Enfermería es ser gerente o gestor en la administración del Cuidado directo y en la solución de problemas frente a éste, situación que requiere de vías de comunicación efectivas y capacidad

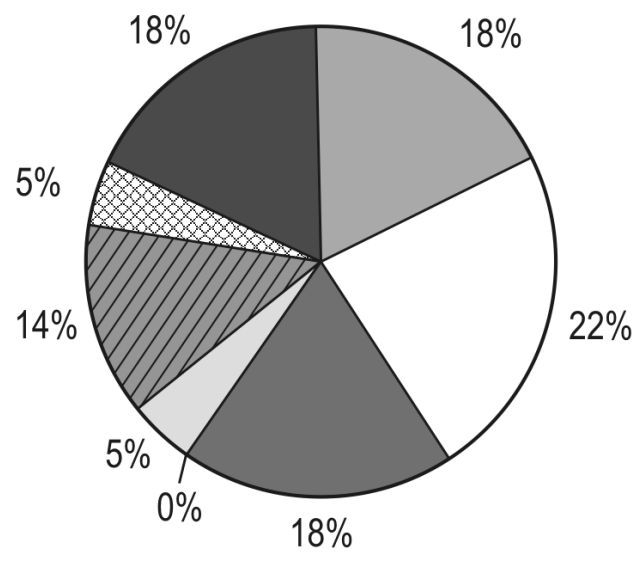

Docente-Estudiante

Docente-Estudiante-Jefe de Servicio.

Docente-Estudiante-Jefe de Servicio-Jefe del Departamento.

Docente-Estudiante-Jefe de Servicio-Jefe del Departamento - Coordinador del servicio.

Docente-Estudiante-Jefe de Servicio-Jefe del Departamento - Coordinador del servicio.

Docente-Estudiante-Jefe de Servicio-Jefe del Departamento - Coordinador del servicio-Institución de Salud.

Docente-Estudiante-Jefe de Servicio-Jefe del Departamento - Coordinador del servicio-Institución de Salud- Institución Universitaria.

No Aplica

Gráfica 2. Instancias utilizadas en la solución del evento. 
para dilucidar las situaciones que se generan por falta de coordinación en el trabajo en equipo.

Un profesional de Enfermería debe planificar, organizar, dirigir y controlar los recursos y el talento humano, con la intención de cumplir, eficazmente, las metas de enfermería en las Instituciones y, como profesional participante, dentro de un equipo de salud, organizar a sus colaboradores, desempeñando el liderazgo en la gestión de un Cuidado de alta calidad, ejercido a conciencia y con responsabilidad (Zárate, 2004).

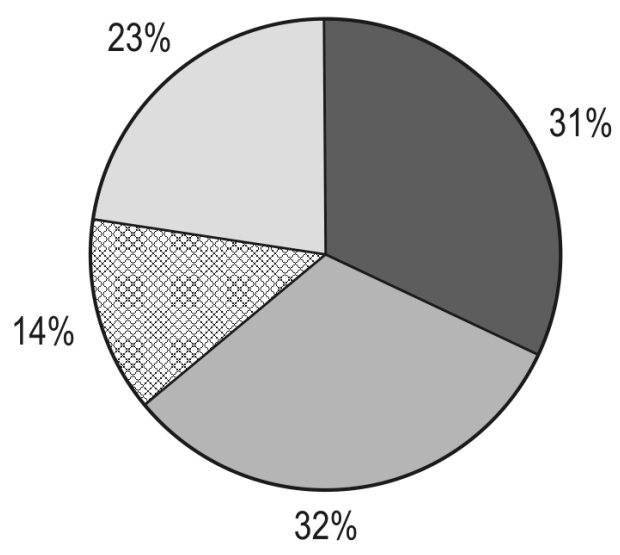

Al analizar los sentimientos que rodearon la situación, se evidenciaron:

a.- Injusticia: se incluyeron sentimientos, como inconformidad e impotencia.

b.- Estrés: se agruparon sentimientos de miedo, de temor, de angustia, de ansiedad y de mal genio.

c.- Sensación de culpa, que incluye la tristeza.

Un porcentaje de entrevistados no expresaron sus sentimientos alrededor del suceso (Gráfica 3).

Gráfica 3. Sentimientos que rodearon el suceso.

El sentimiento de culpabilidad suscitado por el evento, se relacionó con errores cometidos en la realización de procedimientos o en la decisión tomada frente a la atención del Sujeto de Cuidado. En este caso, lo que se puso en juego para el profesional de enfermería fue el dilema ético frente a las decisiones tomadas con el Sujeto de Cuidado.

Los actores implicados en los dilemas éticos realizan una interpretación del suceso que está determinado por el grado de participación en el evento y por sus concepciones frente al Cuidado de Enfermería. Es así como cada individuo va formando la conciencia ética, capacidad que tiene un individuo de reflexionar, de razonar y de hacer una autoexploración de sí mismo y su entorno, con el propósito de orientar su conducta humana, para el ejercicio responsable de la libertad personal (Martínez, 2008).

La responsabilidad de Enfermería lleva implícito el sentido moral cuando las consecuencias de los actos dejan satisfacción personal por el deber cumplido y/o cargos de conciencia por errores en el hacer. Si bien, la actitud positiva hace parte de la responsabilidad moral, no puede ser lo esencial, también es necesario conocer las consecuencias de los actos cuando éstos no son satisfactorios (Zapata, 1992).

La moral de los cuidadores incluye el respeto a los derechos, la integridad, la autonomía y el consentimiento de los individuos, así como la equidad en los servicios, elementos fundamentales de la moral y de la responsabilidad profesional.

Las consecuencias de las decisiones tomadas frente al Cuidado del sujeto llevaron a los profesionales de Enfermería y a los estudiantes en formación a reflexionar sobre los sucesos acontecidos y a reconocer la necesidad de delimitar las responsabilidades del equipo de enfermería, cuando se encuentran estudiantes en rotación (Gráfica 4). 


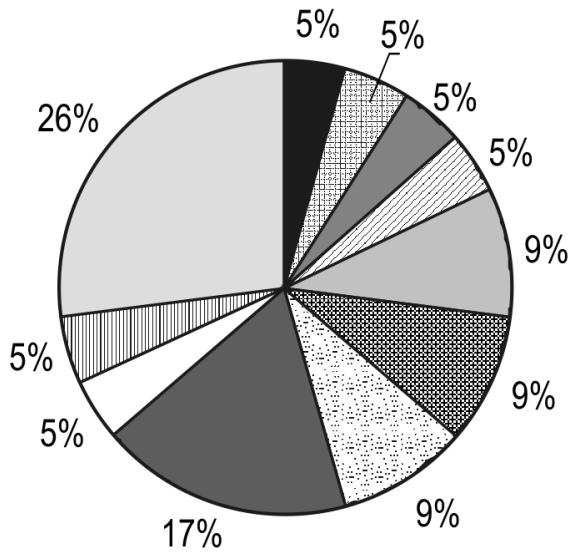

Seguimiento del paciente

Seguimiento de protocolos y del paciente

Llamado de atención del estudiante

Suspensión de la práctica del estudiante

Reflexión del suceso

Análisis de la responsabilidad individual y de lequipo.

Situación sin resolver

No contesta

No aplica

Elaboración de un manual de funciones para Enfermería.

Decisión Médica

Gráfica 4. Decisiones tomadas frente a la solución del evento.

A pesar que se presentaron eventos de riesgo para los Sujetos de Cuidado, ninguno de los profesionales encuestados reportó consecuencias graves para el sujeto que llevaran al profesional de Enfermería de la Institución, al estudiante o al docente supervisor de prácticas a afrontar implicaciones legales.

En la última parte de la encuesta, los docentes supervisores de las prácticas clínicas realizaron las siguientes propuestas, con el fin de evitar incurrir en este tipo de implicaciones. Estas son:

- Establecer las funciones y actividades específicas para los estudiantes y otros miembros del equipo de Enfermería, en las áreas donde realizan su práctica clínica.

- Las Instituciones Prestadoras de Salud deben garantizar las condiciones físicas y de recursos para brindar un Cuidado eficiente y oportuno a los Sujetos de Atención.

- Realizar las acciones de Cuidado directo con calma, verificando siempre cada uno de los procedimientos a realizar.

- Realizar un manual de convivencia entre las dos Instituciones, donde se establezcan los deberes y los derechos de las partes.

- Que la Institución educativa, a través de sus docentes, se involucre en los Comités de Ética Hospitalaria, para nutrirlos con metodologías de resolución de dilemas éticos.
Dados los resultados obtenidos es conveniente enfatizar en la responsabilidad de incrementar en los planes de estudio, los conocimientos en ética y bioética, para que el estudiante, a lo largo de su proceso de formación, vaya desarrollando habilidades cognitivas, con un juicio de valores y destrezas acordes a cada situación, respondiendo al rol que desempeñe y de acuerdo al contexto en el que se desenvuelva (Lombana, 1987).

Los programas de Enfermería conscientes de esta situación han propuesto, dentro de su plan de estudios, la Ética como una asignatura que brinda un marco de referencia para la toma decisiones frente a los dilemas éticos a los que se enfrenta en su cotidianidad; para los enfermeros, específicamente, decisiones frente al Cuidado.

\section{CONCLUSIONES}

En un gran porcentaje, las implicaciones éticas se relacionaron con dilemas de tipo disciplinar frente a decisiones tomadas sobre el Cuidado directo del paciente y la adecuada supervisión del estudiante; otras, se relacionaron con la administración del Cuidado, destacándose como causa, la carencia de recursos materiales y de talento humano en las Instituciones Prestadoras de Salud, para brindar un Cuidado eficiente al individuo. 
Para el profesional que labora en las Instituciones prestadoras de salud, los estudiantes y el docente que supervisa las prácticas, se convierten en un apoyo para la realización de las actividades de Cuidado, dada la cantidad de responsabilidades, de tipo administrativo, que deben asumir y que en ocasiones les impide realizar actividades de Cuidado directo.

Los profesionales docentes que realizan sus prácticas en Instituciones Prestadoras de Salud corren el riesgo de situaciones que los lleven a problemas éticos-legales, cuando no son claras las responsabilidades de cada miembro del equipo de salud y cuando no se cuentan con los materiales requeridos para la atención.

Para el cumplimiento de las competencias de los estudiantes en formación y para disminuir el posible riesgo de cometer un error es necesaria la supervisión directa y continúa del docente, por tanto, el número de estudiantes no debe sobrepasar la capacidad del docente, para realizar la supervisión.

El número de estudiantes debe ser acordado de manera conjunta entre las Instituciones, de tal forma, que no interfiera con el desarrollo normal de las actividades dentro de la Institución.

Las diferentes situaciones que vive el estudiante con el Sujeto de Cuidado, le generan experiencia en el razonamiento moral y en la importancia de los valores para la toma de decisiones éticas.

Determinar las funciones del profesional de Enfermería en las áreas donde se realizan prácticas hospitalarias y estar capacitados en la resolución de conflictos éticos y morales evitará riesgos en el quehacer diario de la profesión, pues la razón de ser la Enfermería, es el Cuidado directo y humanizado de las personas, conservando siempre la dignidad e integridad humana.

El fortalecimiento del Comité Docencia Servicio y el Comité de Ética con la participación de directivos, docentes, personal de los servicios y un representante estudiantil permitirá la construcción de conocimiento, que impulse la investigación desde la mirada de una práctica reflexiva.

\section{BIBLIOGRAFÍA}

ACOFAEN-ALADEFE. 2005. Ética y Enfermería. Boletín Latinoamericano (Colombia). 9(11):1-8.

FRY, S. 1994. La ética en la práctica de Enfermería. Guía para la toma de decisiones éticas. Consejo Internacional de Enfermería. (Ginebra-Suiza). 350p.

LEY 266 de 1996.

LEY 911 de 2004.

LOMBANA C., C. 1987. Experiencias de Ética en enfermería. Revista Avances en Enfermería. 5(1)99-101.

MARTÍNEZ, M. 2008. Conciencia Ética. Instituto Latinoamericano de la comunicación educativa. Centro de estudios en comunicación y tecnologías educativas. México. p.6. http: // www.tuxtepecaldia. com (con acceso 03/09).

PERRY, A.; POTTER, P. 2002. Fundamentos de Enfermería. Ed. Harcourt Océano BarcelonaEspaña. p 387,423 y 425.

ZAPATA V., A. 1992. Construcción de la ética profesional en enfermería. Revista Investigación y educación en Enfermería. (Colombia). 10(2):57-61.

ZÁRATE, R. 2004. La gestión del cuidado de enfermería. Revista Index de Enfermería. (España). 13(4445):1-8.

Recibido: Junio 6 de 2007

Aceptado: Abril 28 de 2009 